\title{
ASYMPTOTIC CONDITIONS FOR PERIODIC SOLUTIONS OF ORDINARY DIFFERENTIAL EQUATIONS
}

\author{
JAMES R. WARD, JR.
}

\begin{abstract}
We obtain sufficient conditions for the existence of periodic solutions to differential equations of the form

$$
x^{(m)}+a_{m-1} x^{(m-1)}+\cdots+a_{1} x^{\prime}+g(t, x)=f(t) \quad(m>1) .
$$

The conditions are necessary for some classes of functions, and require no growth condition on $g(t, x)$ for $x>0(x<0)$.
\end{abstract}

1. Introduction. We consider here ordinary differential equations of the form

$$
x^{(m)}+a_{m-1} x^{(m-1)}+\cdots+a_{1} x^{\prime}+g(t, x)=f(t) \quad(m>1) .
$$

Our purpose is to give a new sufficient condition for the existence of periodic solutions of (1.1). We include the possibility that $g(t, x)$ is bounded below by some constant but not above. We use asymptotic conditions on $g(t, x)$ as $x \rightarrow \pm \infty$ (but not on $\left.g(t, x) x^{-1}\right)$. For some classes of nonlinearities our asymptotic conditions are also necessary. Our methods also apply in case $g$ grows slowly at $-\infty$ or depends on the derivatives of $x$ up to $x^{(m-1)}$, or if $g$ is bounded above but not below. The class of $g$ 's which we consider include $g(t, x)=p(t) e^{x}$ with $p \geqslant 0$ (or $\leqslant 0$ ).

Our results are related to some of those announced by Dancer [1], to those of Fucik and Lovicar [2], Gupta [3], Lazer [4], Mawhin [5], Mawhin and Schmitt [7], and Reissig [8]. Our results are in substance (but not in method) related to some of those announced in [1]. Here we allow the nonlinearity to depend on $t$ and the derivatives of $x$ and do not need the regularity assumptions made on the nonlinearity in the super linear case in [1], nor do we need uniqueness for initial value problems. Moreover our results apply to equations of arbitrary order. The papers [1]-[5], [7], and [8] all use assumptions on the growth of the nonlinearity at each of $\pm \infty$. Here we make no assumption on the growth of $g(t, x)$ for $x>0$ (or else none for $x \leqslant 0$ ) and this is perhaps the most interesting aspect of our results. In the special case of $m=1$ in equation (1.1) Mawhin in [6, p. 71] has obtained results similar to ours without our condition (c2) of Theorem 1. Our results are in fact a partial generalization to higher order equations the existence result of $[6, p .71]$. The method of obtaining a priori bounds for the solutions in [6, p. 71] is however specific to the case of a first order equation.

Received by the editors February 28, 1980.

1980 Mathematics Subject Classification. Primary 34C15, 34C25.

Key words and phrases. Periodic solutions, nonlinear equations, coincidence degree. 
Our proof makes use of an abstract continuation theorem of Mawhin. For the convenience of the reader we state this theorem in $\$ 2$, and in $\$ 3$ we state and prove our theorem.

2. Preliminaries. Let $X$ and $Z$ be normed vector spaces, $L: D(L) \subseteq X \rightarrow Z$ a linear Fredholm mapping of index zero (index $(L)=\operatorname{dim} \operatorname{ker}(L)-\operatorname{codim} \operatorname{Im}(L)$ ) and $N: X \rightarrow Z$ a continuous mapping. It follows that there exist continuous projections $P: X \rightarrow X$ and $Q: Z \rightarrow Z$ such that $\operatorname{Im}(P)=\operatorname{ker}(L)$ and $\operatorname{Im}(L)=$ $\operatorname{ker}(Q)=\operatorname{Im}(I-Q)$. Moreover the mapping $L: D(L) \cap \operatorname{ker}(P) \rightarrow \operatorname{Im}(L)$ is invertible; denote its inverse by $K$. Let $\Omega$ be an open bounded subset of $X$. The mapping $N$ is said to be $L$-compact on $\bar{\Omega}$ if $Q N(\bar{\Omega})$ is bounded and $K(I-Q) N: \bar{\Omega} \rightarrow X$ is compact. Let $J$ be an isomorphism from $\operatorname{Im}(Q)$ onto $\operatorname{ker}(L)$; such a $J$ exists since these subspaces have the same finite dimension.

Theorem A (MAWHIN [5], [6]). Let L be a Fredholm mapping of index zero and let $N$ be L-compact on $\bar{\Omega}$. Suppose

(i) for each $\lambda \in(0,1)$ and each $x \in \operatorname{Dom} L \cap \partial \Omega, L x+\lambda N x \neq 0$,

(ii) $Q N x \neq 0$ for each $x \in \operatorname{ker} L \cap \partial \Omega$ and $d(J Q N, \Omega \cap \operatorname{ker} L, 0) \neq 0$.

Then the equation $L x+N x=0$ has at least one solution in $D(L) \cap \bar{\Omega}$.

Here $d(\cdot, \cdot, \cdot)$ refers to the Brouwer degree.

In what follows $C_{T}^{0}$ will denote the linear space of real valued continuous $T$-periodic functions on $R=(-\infty, \infty)$. The linear space $C_{T}^{0}$ is a Banach space with the usual norm for $u \in C_{T}^{0}$ given by $|u|_{0}=\max _{t \in R}|u(t)|$. By $C_{T}^{m}(m>1)$ we denote the linear space of $T$-periodic functions with $m$ continuous derivatives. $C_{T}^{m}$ is a Banach space with norm $|x|_{m}=\max \left\{\left|x^{(i)}\right|_{0}: 0<i<m\right\}$.

3. Main results. Let $g: R \times R \rightarrow R$ be continuous and $T$-periodic $(T>0)$ in its first variable; i.e., $g(t+T, x)=g(t, x)$ for all $t, x$. We define two measurable functions $\mu_{+}, \mu_{-}: R \rightarrow R \cup\{-\infty, \infty\}$ by

$$
\begin{array}{ll}
\mu_{+}(t)=\liminf _{x \rightarrow \infty} g(t, x), & t \in R, \\
\mu_{-}(t)=\limsup _{x \rightarrow-\infty} g(t, x), & t \in R .
\end{array}
$$

We assume $g(t, x)$ is bounded below for $x>0$ and bounded above for $x<0$. More precisely, we assume there are constants $\delta_{+}$and $\delta_{-}$with $g(t, x)>\delta_{+}$for $x \geqslant 0$ and $g(t, x) \leqslant \delta_{-}$for $x \leqslant 0$.

Let $a_{1}, \ldots, a_{m-1}$ be real constants. We consider the equation (1.1) with $f \in C_{T}^{0}$. In the following, for $x \in C^{m}$, we let

$$
L x \equiv x^{(m)}+a_{m-1} x^{(m-1)}+\cdots+a_{1} x^{\prime} .
$$

TheOrem 1. Assume:

(c1) The only $T$-periodic solutions to the equation $L x=0$ are the constants.

(c2) There are numbers $\alpha$ and $\beta$ such that for all $(t, x) \in R \times R,|g(t, x)|<$ $g(t, x)+\alpha|x|+\beta$.

(c3) $\int_{0}^{T} \mu_{-}(t) d t<\int_{0}^{T} f(t) d t<\int_{0}^{T} \mu_{+}(t) d t$.

Then there is a number $\alpha_{0}>0$ such that equation (1.1) has a T-periodic solution provided $\alpha<\alpha_{0}$. 
REMARKs. In (c3) the integral on the left may be $-\infty$ and the integral on the right may be $+\infty$. A dual theorem is also true with $\mu_{+}(t)=\lim _{\inf _{x \rightarrow-\infty}} g(t, x), \mu_{-}(t)=$ lim $\sup _{x \rightarrow \infty} g(t, x)$, and (c3) holding. Also, if (c2) is replaced by $|g(t, x)|<-g(t, x)$ $+\alpha|x|+\beta$, the same conclusions hold. Note that if $g(t, x)$ is a uniformly bounded function on $R \times R$ then it will satisfy (c2).

Before proving Theorem 1, we will prove the following lemma.

Lemma 1. Suppose that (c1) holds. Then there is a number $\mu>0$ such that

$$
\mu \int_{0}^{T}\left|x^{(m)}\right| d t<\int_{0}^{T}|L x| d t \quad \text { for all } x \in C_{T}^{m} .
$$

Proof. Suppose (3.1) fails to hold. Then for each positive integer $n$ there is a function $x_{n} \in C_{T}^{m}$ with $\int_{0}^{T} x_{n} d t=0$ and

$$
\int_{0}^{T}\left|L x_{n}\right| d t<\frac{1}{n} \int_{0}^{T}\left|x_{n}^{(m)}\right| d t
$$

Since we may divide each side of (3.2) by

$$
\left\|x_{n}\right\|_{m} \equiv\left|x_{n}\right|_{m-1}+\int_{0}^{T}\left|x_{n}^{(m)}\right| d t
$$

we may assume that $\left\|x_{n}\right\|_{m}=1$ and

$$
\int_{0}^{T}\left|L x_{n}\right| d t<\frac{1}{n}
$$

The set $\left\{x_{n}\right\}_{n=1}^{\infty}$ is precompact in $C_{T}^{m-1}$ and hence has a subsequence which we relabel as $\left\{x_{n}\right\}_{n=1}^{\infty}$, which converges in $C_{T}^{m-1}$ to a function $\tilde{x} \in C_{T}^{m-1}$. By (3.3),

$$
\lim _{n \rightarrow \infty} \int_{0}^{T}\left|L x_{n}\right| d t=0
$$

Hence $\left\{x_{n}^{(m)}\right\}_{n=1}^{\infty}$ converges in $L^{1}(0, T)$ to $-\left(a_{m-1} \tilde{x}^{(m-1)}+\cdots+a_{1} \tilde{x}^{\prime}\right)$. Therefore, since

$$
x_{n}^{(m-1)}(t)=x_{n}^{(m-1)}(0)+\int_{0}^{t} x_{n}^{(m)}(s) d s
$$

for $n=1,2, \ldots$, taking limits we conclude that $\tilde{x} \in C_{T}^{m}$ and $L \tilde{x}=0$. Thus $\tilde{x}$ is a constant function. Moreover, since $\int_{0}^{T} x_{n} d t=0$ for all $n, \tilde{x}$ also has zero mean value, and we have $\tilde{x} \equiv 0$. But $\left\|x_{n}\right\|_{m}=1$ implies $\|\tilde{x}\|_{m}=1$, and this contradiction proves the lemma.

Proof of Theorem 1. We will apply Mawhin's theorem (\$2). Let

$$
X=Z=C_{T}^{0}, \quad D(L)=C_{T}^{m}, \quad L: u \mapsto L u \quad \text { for } u \in D(L) .
$$

Define $N: X \rightarrow X$ by $N u(t)=g(t, u(t))-f(t)$ for $u \in X$ and $t \in R$. Now $x \in C_{T}^{m}$ is a $T$-periodic solution of (1.1) if and only if $L x+N x=0$.

It is well known that $L$ is a Fredholm operator of index zero. In fact for $u \in X=Z$ we define

$$
P u=Q u=\frac{1}{T} \int_{0}^{T} u(s) d s .
$$

Then $\operatorname{ker}(L)=\operatorname{Im}(P)=R$ and $\operatorname{Im}(L)=\operatorname{ker}(P)=\operatorname{Im}(I-P)$, so that $\operatorname{Im}(L)$ is closed and $\operatorname{dim} \operatorname{ker}(L)=1$ and $\operatorname{codim} \operatorname{Im}(L)=1$ also. Thus the mapping $J$ may be 
chosen to be the identity on $R$. Moreover it is easily verified that

$$
K=(L \mid \operatorname{ker} P \cap \operatorname{Dom} L)^{-1}
$$

is a compact mapping from $\operatorname{Im}(I-P)$ onto $\operatorname{Dom}(L)=D(L)$. It is well known that $N$ maps $X$ into itself continuously, and takes bounded sets into bounded sets. It follows that $K(I-P) N$ is a completely continuous self-map of $X$, and $P N$ maps bounded sets in $X$ into bounded sets. Now we consider the family of equations

$$
L x+\lambda N x=0, \quad 0<\lambda<1,
$$

and we will show the existence of a number $p_{0}>0$ such that if $|x|_{0}>p_{0}$ then $L x+\lambda N x \neq 0,0<\lambda<1$.

For any solution $x$ of (3.4) we have $L x=-\lambda g(t, x)+\lambda f(t)$ and thus by (c2)

$$
|L x(t)| \leqslant g(t, x)+\alpha|x|+\beta+|f|_{0} .
$$

Integrating (3.5) from 0 to $T$ and observing that, by the periodicity of $x$ and its derivatives, we must have $\int_{0}^{T} g(t, x(t)) d t=\int_{0}^{T} f(t) d t$, we obtain

$$
\int_{0}^{T}|L x| d t \leqslant \alpha T|x|_{0}+\left(2|f|_{0}+\beta\right) T
$$

By Lemma 1 we have

$$
\int_{0}^{T}\left|x^{(m)}\right| d t \leqslant \alpha T \mu^{-1}|x|_{0}+\left(2|f|_{0}+\beta\right) T \mu^{-1} .
$$

Since $x$ is $T$-periodic there are numbers $z_{1}, \ldots, z_{m-1}$ in $[0, T]$ with $x^{(k)}\left(z_{k}\right)=0$, $1 \leqslant k \leqslant m-1$. Using (3.6) and $x^{(k)}(t)=\int_{z_{k}}^{t} x^{(k-1)}(s) d s$ we obtain

$$
\left|x^{(k)}\right|_{0} \leqslant \alpha T^{m-k} \mu^{-1}|x|_{0}+C_{k}, \quad 1 \leqslant k \leqslant m-1,
$$

where $C_{k}=T^{m-k} \mu^{-1}\left(2|f|_{0}+\beta\right)$.

We will now show that there is a number $r_{0}>0$ such that for each solution $x$ to (3.4) there is a number $z \in[0, T]$ with $|x(z)|<r_{0}$. Suppose that for each positive integer $n$ there is a $\lambda_{n} \in(0,1)$ and a solution $x_{n}$ of $L x+\lambda_{n} N x=0$ with $x_{n}(s) \geq n$ for all $s \in R$. Then integrating

$$
L x_{n}+\lambda_{n} g\left(t, x_{n}\right)=\lambda_{n} f(t)
$$

from 0 to $T$ and using the periodicity of $x_{n}$, we obtain

$$
\int_{0}^{T} g\left(t, x_{n}(t)\right) d t=\int_{0}^{T} f(t) d t
$$

But $\lim \inf _{n \rightarrow \infty} g\left(t, x_{n}(t)\right) \geqslant \mu_{+}(t)$.

By Fatou's lemma (here we use $g(t, x) \geqslant \delta_{+}$for all $x \geqslant 0$ ) we have

$$
\int_{0}^{T} f(t) d t=\liminf _{n \rightarrow \infty} \int_{0}^{T} g\left(t, x_{n}(t)\right) d t>\int_{0}^{T} \mu_{+}(t) d t
$$

which contradicts (c3). Thus there is a number $r_{1}>0$ such that if $x$ is a solution of (3.4) for any $0<\lambda<1$ there is a number $s_{1} \in[0, T]$ with $x\left(s_{1}\right)<r_{1}$. Similarly by using $\mu_{-}$and Fatou's lemma one may show the existence of a number $r_{2}>0$ such that for any solution $x$ there is a number $s_{2} \in[0, T]$ with $x\left(s_{2}\right)>-r_{2}$. By continuity of $x$ there is a $z \in[0, T]$ with $|x(z)| \leqslant r_{0}, r_{0}=\max \left\{r_{1}, r_{2}\right\}$.

Using (3.7) we now have from $x(t)=\int_{z}^{t} x^{\prime}(s) d s+x(z)$ that $|x|_{0} \leqslant r_{0}+C_{1} T+$ $\alpha T^{m} \mu^{-1}|x|_{0}$ 
Thus if $0 \leqslant \alpha<\alpha_{0}=\mu T^{-m}$ then

$$
|x|_{0} \leqslant\left(1-\alpha T^{m} \mu^{-1}\right)^{-1}\left(r_{0}+C_{1} T\right) \equiv p_{0} .
$$

If $r>p_{0}$ then (3.4) has no solutions with $|u|_{0}>r$. This verifies (i) of Mawhin's theorem for any

$$
\Omega=\Omega_{r}=\left\{u \in C_{T}^{0}:|u|_{0}<r\right\} \quad \text { if } r>p_{0} .
$$

We now verify (ii) of Mawhin's theorem. Let $x \in \operatorname{ker} L \cap \partial \Omega_{r}$; then $x \in R$ and $x= \pm r$. We have

$$
Q N x=\frac{1}{T} \int_{0}^{T} g(s, x) d s-\frac{1}{T} \int_{0}^{T} f(s) d s .
$$

By (c3) and two more applications of Fatou's lemma we have the existence of a $p_{1}>0$ such that $r>p_{1}$ implies $Q N(r)>0$ and $Q N(-r)<0$. Let $d=\max \left\{p_{0}, p_{1}\right\}$ +1 , and $\Omega=\left\{u \in C_{T}^{0}:|u|_{0}<d\right\}$. We have $L x+\lambda N x \neq 0$ for $0<\lambda<1$ and $x \in \partial \Omega, Q N x \neq 0$ for $x \in \operatorname{ker} L \cap \partial \Omega$, and $d(Q N$, $\operatorname{ker} L \cap \partial \Omega, 0) \neq 0$. By Mawhin's theorem there is a solution to the equation $L x+N x=0$ in $\bar{\Omega}$. That is, equation (1.1) has a $T$-periodic solution.

EXAMPLE. Let $a_{1}, \ldots, a_{m-1}$ be real numbers such that the equation $r^{m-1}+$ $a_{m-1} r^{m-2}+\cdots+a_{1}=0$ has no roots of the form $2 n \pi i / T, n$ an integer, $n \neq 0$. Then the equation $x^{(m)}+a_{m-1} x^{(m-1)}+\cdots+a_{1} x^{\prime}=0$ has no $T$-periodic solutions except the constants. By Theorem 1 the equation

$$
x^{(m)}+a_{m-1} x^{(m-1)}+\cdots+a_{1} x^{\prime}+p(t) e^{x}=f(t)
$$

has a $T$-periodic solution if $p, f$ are continuous $T$-periodic functions with $p>0$ $(\leqslant 0), p \neq 0$, and $\int_{0}^{T} f>0(<0)$.

Extensions. One could assume only Carathéodory conditions on $g$ and $f$ and obtain similar results.

If $g(t, x)$ in $(1.1)$ is replaced by $g\left(t, x, x^{\prime}, \ldots, x^{(m-1)}\right)$ similar results can be proven. Assume the existence of functions $\mu_{ \pm}$satisfying

$$
\mu_{+}(t) \leqslant \liminf _{x \rightarrow \infty} g\left(t, x, y_{1}, \ldots, y_{m-1}\right)
$$

and

$$
\mu_{-}(t) \geqslant \limsup _{x \rightarrow-\infty} g\left(t, x, y_{1}, \ldots, y_{m-1}\right)
$$

uniformly for $\left(y_{1}, \ldots, y_{m-1}\right)$ in compact subsets of $R^{m-1}$. Assume the existence of nonnegative numbers $\alpha_{0}, \ldots, \alpha_{m-1}, \beta$ such that

$$
\begin{aligned}
\left|g\left(t, x, y_{1}, \ldots, y_{m-1}\right)\right| \leqslant & g\left(t, x, y_{1}, \ldots, y_{m-1}\right) \\
& +\alpha_{0}|x|+\alpha_{1}\left|y_{1}\right|+\cdots+\alpha_{m-1}\left|y_{m-1}\right|+\beta .
\end{aligned}
$$

If the other hypotheses of Theorem 1 now hold, there is a number $\varepsilon_{0}>0$ such that this new problem has a $T$-periodic solution if $\max \left\{\alpha_{i}: 0<i<m-1\right\}<\varepsilon_{0}$. The proof is much the same as that of Theorem 1 .

If $g(t, x)$ is strictly increasing in $x$ for fixed $t$ then (c3) can be seen to be necessary for a $T$-periodic solution. 
Theorem 1 could also be extended to $n$-dimensional vector differential equations analogous to (1.1) by making hypotheses such as those of Theorem 7.1 of [5] on the linear part of the equation and appropriate hypotheses on the components $g_{i}(t, x)$ of the nonlinear part $g(t, x)=\left(g_{i}(t, x)\right)$.

\section{REFERENCES}

1. E. N. Dancer, Boundary value problems for weakly nonlinear ordinary differential equations, Bull. Austral. Math. Soc. 15 (1976), 321-328.

2. S. Fucik and V. Lovicar, Periodic solutions of the equation $x^{\prime \prime}(t)+g(x(t))=p(t)$, Casopis Pèst. Mat. 100 (1975), 160-175.

3. C. P. Gupta, On functional equations of Fredholm and Hammerstein type with applications to existence of periodic solutions of certain ordinary differential equations, J. Integral Equations (to appear).

4. A. C. Lazer, On Schauder's fixed point theorem and forced second-order nonlinear oscillations, J. Math. Anal. Appl. 21 (1968), 421-425.

5. J. Mawhin, Periodic solutions of some vector retarded functional differential equations, J. Math. Anal. Appl. 45 (1974), 588-603.

6. Topological degree methods in nonlinear boundary value problems, CBMS Regional Conf. Ser. in Math., no. 40, Amer. Math. Soc. Providence, R.I., 1979.

7. J. Mawhin and K. Schmitt, Rothe and Altman type coincidence theorems and applications to differential equations, Nonlinear Anal. 1 (1977), 151-160.

8. R. Reissig, Periodic solutions of certain higher order differential equations, Nonlinear Anal. 2 (1978), 635-642.

Department of Mathematics, University of Alabama, University, Alabama 35486 